Pacific

Journal of

Mathematics

ZEROS OF FAMILIES OF AUTOMORPHIC L-FUNCTIONS CLOSE TO 1

E. Kowalski AND P. Michel

Volume $207 \quad$ No. 2

December 2002 


\title{
ZEROS OF FAMILIES OF AUTOMORPHIC $L$-FUNCTIONS CLOSE TO 1
}

\author{
E. Kowalski AND P. Michel
}

\begin{abstract}
For many $\boldsymbol{L}$-functions of arithmetic interest, the values on or close to the edge of the region of absolute convergence are of great importance, as shown for instance by the proof of the Prime Number Theorem (equivalent to non-vanishing of $\zeta(s)$ for mathrmRe $(s)=1)$. Other examples are the Dirichlet $L$-functions (e.g., because of the Dirichlet class-number formula) and the symmetric square $L$-functions of classical automorphic forms. For analytic purposes, in the absence of the Generalized Riemann Hypothesis, it is very useful to have an upper-bound, on average, for the number of zeros of the $L$-functions which are very close to 1 . We prove a very general statement of this type for forms on $G L(n) / Q$ for any $n \geqslant 1$, comparable to the log-free density theorems for Dirichlet characters first proved by Linnik.
\end{abstract}

\section{Introduction.}

The old preprint [KM1] contained a few density results for the zeros of $L$-functions attached to modular forms, which were not included in the published version [KM2]. Here we partly correct this state of affairs by presenting an extension of one of those results, namely [KM1, Théorème 1.2]. The extension consists in treating much more general families of automorphic $L$-functions on $G L(n), n \geqslant 1$, in the spirit of [DK, $\S 4]$ for instance. That this is possible is because we study zeros of $L$-functions close to the edge of the critical strip, and in such a region everything behaves very much as if all the $L$-functions involved were of degree 1 (compare for instance [KM2, §3]). One point worth mentioning, however, is that we avoid using the analogue of the Hoffstein-Lockhart bound, which would not be available in general (compare [KM1, 8.3, p. 38]).

We now state the theorem. Let $n \geqslant 1$ be a fixed integer. We assume we are given, for all $q \geqslant 1$, a finite set (possibly empty) $S(q)$ of cuspidal automorphic representations $^{1}$ of $G L(n) / \mathbf{Q}([\mathbf{B u}],[\mathbf{B C}])$ which satisfy the following conditions:

${ }^{1}$ This means in particular irreducible for us. 
(1) The forms $f \in S(q)$ satisfy the Ramanujan-Petersson conjecture at the finite places, namely all the local components $f_{p}$ of $f$ at a prime $p$ are tempered.

(2) There exists $A>0$ such that for all $f \in S(q)$, the conductor ([JPS]) Cond $(f)$ of $f$ satisfies

$$
\operatorname{Cond}(f) \leqslant q^{A} \text {. }
$$

(3) There exists $d>0$ such that

$$
|S(q)| \ll q^{d}
$$

for all $q \geqslant 1$, the implied constant depending only on the family.

(4) All the $f \in S(q)$ have the same component at $\infty$, hence the same gamma factor in the functional equation.

The data of all $S(q), q \geqslant 1$, is called here a family of automorphic forms; in particular, if we speak of a constant depending only on the family, it means independent of $q$ (and in general will depend only on $A, d$ and the common gamma factor of $f \in S(q)$ ). We will give below various examples of such families and comment on the assumptions.

Remark 1. We will use the Ramanujan-Petersson Conjecture in its form for local $L$-functions: If $p$ is unramified for $f$, then $f_{p}$ being tempered is equivalent with the condition that the "local roots" of $L_{p}(f)$ (the Satake parameters) $\alpha_{i, p}$ satisfy

$$
\left|\alpha_{i, p}\right|=1 \text {. }
$$

For $p$ a ramified prime, we will only use the consequence of temperedness that the local $L$-function $L_{p}(f, s)$ is holomorphic for $\operatorname{Re}(s)>0$ (see e.g., [RS, App. A]). ${ }^{2}$

Now we let

$$
M(\alpha, T)=\{z \in \mathbf{C} \mid \operatorname{Re}(z) \geqslant \alpha, \text { and }|\operatorname{Im}(z)| \leqslant T\}
$$

for $\alpha \in \mathbf{R}, T \geqslant 0$. For any cuspidal automorphic representation $f$ on $G L(n) / \mathbf{Q}$, we let

$$
N(f ; \alpha, T)=|\{\rho \in M(\alpha, T) \mid L(f, \rho)=0\}|
$$

(zeros counted with multiplicity).

Our main theorem is the following.

${ }^{2}$ Being holomorphic for $\operatorname{Re}(s)>1 / 2-\delta$ for some $\delta>0$ depending only on the family would suffice for our application and this is known in general [RS, Prop. A1]. 
Theorem 2. Let $S(q), q \geqslant 1$, be as above. Let $\alpha \geqslant 3 / 4$ and $T \geqslant 2$. Then there exists $c_{0}>0$, depending only on the family, such that

$$
\sum_{f \in S(q)} N(f ; \alpha, T) \ll T^{B} q^{c_{0} \frac{1-\alpha}{2 \alpha-1}}
$$

for all $q \geqslant 1$ and some $B \geqslant 0$ (depending only on the family). The implied constant depends only on the family and the choice of $c_{0}$, and one can choose any $c_{0}>c_{0}^{\prime}$, where

$$
c_{0}^{\prime}=\frac{5 n A}{2}+d .
$$

Remark 3. This theorem is only useful when $\alpha$ is very close to 1 and the $T$-aspect is essentially irrelevant (by smoothing for instance). Indeed we have by classical $L$-function theory

$$
N(f ; 0, T) \sim \frac{n T}{2 \pi} \log (\operatorname{Cond}(f) T) \text { as } T \rightarrow+\infty,
$$

so the estimate (1.4) is nontrivial only for $T$ fixed and

$$
\alpha \geqslant 1-\frac{d}{c_{0}} \text {. }
$$

In that region, it shows that there can only be very few zeros of the $L$ functions $L(f, s)$ on average over $f \in S(q)$.

Remark 4. One can weaken the assumption that the Ramanujan-Petersson conjecture holds, and use an estimate towards it of the form

$$
\left|\alpha_{i, p}\right| \leqslant p^{\theta}
$$

for some $\theta$ with $0<\theta<1 / 4$ (depending only on the family). The requirement $\theta<1 / 4$ comes from Lemma 9 below to get a factorization of the Rankin-Selberg convolution valid in $\operatorname{Re}(s)>1-\gamma$ for some $\gamma>0$ depending only on the family.

In particular this is known in full generality for $G L(2)$ over a number field, where one can take, e.g., $\theta=7 / 64$ (Kim and Sarnak [KS]). For $n \geqslant 3$, the best general bounds are not sufficient. But note that applying the result of [RS, App. A] that $\theta=1 / 2-1 /\left(n^{2}+1\right)$ works for cusp forms on $G L(n)$ shows that a suitable bound will exist whenever a symmetric square lift exists, for instance.

Theorem 5. Let $S(q), q \geqslant 1$, be a family satisfying (1.6). Let $\alpha \geqslant 3 / 4$ and $T \geqslant 2$. Then there exists $c_{0}>0$, depending only on the family, such that

$$
\sum_{f \in S(q)} N(f ; \alpha, T) \ll T^{B} q^{c_{0} \frac{1-\alpha}{2 \alpha-1}}
$$


for all $q \geqslant 1$ and some $B \geqslant 0$ (depending only on the family). The implied constant depends only on the family and the choice of $c_{0}$, and one can choose any $c_{0}>c_{0}^{\prime}$, where

$$
c_{0}^{\prime}= \begin{cases}\frac{5 n A}{2}+d, & \text { if } \theta=0 \\ \frac{2}{1-4 \theta}\left(\frac{n A}{2}+8 n A \vartheta(1+\theta / 2)\right), & \text { if } \theta>0, \text { where } \vartheta=\frac{1+\theta}{2(1+2 \theta)} .\end{cases}
$$

We will not present this computation (see also $[\mathbf{L u}]$ ). Note that the Ramanujan-Petersson conjecture is expected to hold for $G L(n)$, and is known, by work of Eichler-Shimura, Deligne and Deligne-Serre, for classical holomorphic cusp forms, and hence follows for families constructed out of such forms by Langlands functoriality (symmetric square, etc...). Moreover, it also holds for automorphic forms associated to Artin $L$-functions or to algebraic varieties (by Deligne's proof of the Weil Conjectures) as soon as they are known to exist.

Theorem 2 can sometimes replace the Generalized Riemann Hypothesis in applications. The case of Dirichlet characters was indeed established for that purpose by Linnik [Li1], [Li2] who used it as one of the main steps in his proof that the least prime $p \equiv a(\bmod q)$, for $(a, q)=1$, satisfies

$$
p \ll q^{L_{1}}
$$

for some absolute constant $L_{1} \geqslant 0$ (see also e.g., [Bo], [J] for proofs of Linnik's theorem; the best known result is due to Heath-Brown with $L_{1}=5.5$ ). Our proof of Theorem 2 will follow Jutila's version of ideas of Selberg $[\mathbf{J}]$.

Remark 6. Using base-changed $L$-functions from a number field $K$ (when they are known to exist), one can give applications of our theorem along the same lines as Linnik's, concerning the distribution of prime ideals in ray-class groups for instance. However, such a generalization has already been proved in full generality by Fogels $[\mathbf{F o}]$ and (with better uniformity in terms of $K$ ) by Weiss [We].

However we take this opportunity to mention the following nice corollary, which we have not found explicitly stated in the references above:

Corollary 7. There exists an absolute constant $L_{2}>0$, such that for any $n>0$, the smallest prime $p$ of the form $p=x^{2}+n y^{2}$, with $x, y \in \mathbf{Z}$, satisfies

$$
p \ll n^{L_{2}},
$$

with an absolute implied constant.

To prove this, one need only prove that for any imaginary quadratic order $\mathcal{O}$ with discriminant $D$, there is a principal prime ideal $\mathfrak{p}$ in $\mathcal{O}$ of degree 1 with norm $N \mathfrak{p} \ll|D|^{L_{2}}$ for some $L_{2}>0$, and apply this to $\mathcal{O}=\mathbf{Z}[\sqrt{-n}]$. This is a special case of the results of Fogels and Weiss, and can be proved 
using Linnik's method and Theorem 2 applied to the theta functions associated to characters of ray-class groups (see e.g., [Iw1, Ch. 12]).

See Cox's book $[\mathbf{C}]$ for much more about primes of the form $x^{2}+n y^{2}$. It would be interesting to compute an admissible value of $L_{2}$.

Another possible application of this density theorem consists in the asymptotic evaluation of the moments of $L(f, 1)$ for properly chosen families. For $(L(\chi, 1))_{\chi}$, the family of primitive Dirichlet $L$-functions for characters modulo $q$, this is a very classical topic (see $[\mathbf{M V}]$ and the more recent $[\mathbf{G S}]$ for a deep study of the problem). For higher degree $L$-functions, this question was considered recently by Luo $[\mathbf{L u}]$ in the case of symmetric square $L$ functions of Maass forms with large eigenvalues, and by E. Royer [Ro] for the case of symmetric square $L$-functions of holomorphic forms with large level. In his work, Royer used the main result of this paper to obtain a good approximation of $L\left(\operatorname{sym}^{(2)} f, 1\right)^{ \pm 1}$ by a very short Dirichlet polynomial, valid for almost all forms $f$.

In a forthcoming paper, we will use the corollary below to evaluate such moments for algebraic families of $L$-functions (as opposed to the spectral ones given above).

More precisely, we will consider a one parameter family of elliptic curves defined over $\mathbf{Q}$ and given by the equation

$$
E_{t}: y^{2}+a_{1}(t) x y+a_{3}(y)=x^{3}+a_{2}(t) x+a_{4}(t) x+a_{6}(t)
$$

with $a_{i}(t) \in \mathbf{Z}[t]$. We note $\Delta(t) \neq 0$ the discriminant of the generic fiber, and $j(t) \in \mathbf{Q}(t)$ its $j$-invariant.

In the sequel we assume that the family is not geometrically trivial, i.e., $j(t) \notin \mathbf{Q}$. For any $x \in \mathbf{Z}$ such that $\Delta(x) \neq 0$, the specialized fiber $E_{x}$ is an elliptic curve and from the work of Wiles, Taylor-Wiles, Breuil-ConradDiamond-Taylor ([Wi, TW, Di, BCDT $]$ ) and from the existence of the Gelbart-Jacquet lift [GJ], we know that the (analytically normalized) $L$ functions $L\left(E_{x}, s\right), L\left(\operatorname{sym}^{(2)} E_{x}, s\right)$ associated to $E_{x}$ and to its symmetric square come from automorphic forms on $G L_{2}(\mathbf{Q})$ and $G L_{3}(\mathbf{Q})$ respectively.

Denote by $N\left(E_{x} ; \alpha, T\right)$ and $N\left(\operatorname{sym}^{(2)} E_{x} ; \alpha, T\right)$ the number of zeros of these $L$-functions in $M(\alpha, T)$.

We will deduce the following Corollary from Theorem 2: 
Corollary 8. With the above notations (recall that $j(t)$ is not constant), we have

$$
\begin{aligned}
\sum_{\substack{|x| \leqslant X \\
\Delta(x) \neq 0}} N\left(E_{x} ; \alpha, T\right) & \ll T^{B} X^{c_{1} \frac{1-\alpha}{2 \alpha-1}} \\
\sum_{\substack{|x| \leqslant X \\
\Delta(x) \neq 0}} N\left(\operatorname{sym}^{(2)} E_{x} ; \alpha, T\right) \ll T^{B} X^{c_{2} \frac{1-\alpha}{2 \alpha-1}} &
\end{aligned}
$$

for all $X, T \geqslant 1$ and some absolute constant $B \geqslant 0$. The implied constants depends only on the choice of $c_{1}, c_{2}$, and one can choose any $c_{1}>c_{1}^{\prime}$ and $c_{2}>c_{2}^{\prime}$, where

$$
c_{1}^{\prime}=5 \operatorname{deg} \Delta+1, c_{2}^{\prime}=15 \operatorname{deg} \Delta+1 .
$$

This density result will be one of the main tools for the study of the moments of $L\left(E_{x}, 1\right)$ and $L\left(\operatorname{sym}^{(2)} E_{x}, 1\right)$.

We will prove Theorem 2 in Sections 2, 3 and 4 and Corollary 8 in Section 5 .

\section{Factorization of $L$-functions.}

We fix a family $S(q), q \geqslant 1$.

For $f \in S(q)$, we write

$$
L(f, s)=\sum_{n \geqslant 1} \lambda_{f}(n) n^{-s}
$$

the Dirichlet series expansion of its $L$-function (without gamma factor). There are minor annoyances arising from the ramified primes $p \mid \operatorname{Cond}(f)$, as usual. In this paper, the most convenient way to deal with them is to use only the unramified $L$-function. Therefore, in all that follows, we use $L(f, s)$ to denote the unramified $L$-function. This means that

$$
\lambda_{f}(n)=0 \quad \text { if }(n, \operatorname{Cond}(f)) \neq 1 .
$$

Also note that the unramified $L$-function differs from the complete $L$-function by a finite Euler product which is entire, and doesn't vanish in $\operatorname{Re}(s)>0$ (see Remark 1).

The main simplifying fact in considering zeros close to 1 is that in a region $\operatorname{Re}(s)>1 / 2+\delta$, with $\delta>0$, all $L$-functions we consider behave as if they were Euler products of degree 1. This applies not only to $L(f, s)$ for $f \in S(q)$, but also to their Rankin-Selberg convolutions [JPS]. We recall briefly that by work of Jacquet, Piatetskii-Shapiro and Shalika, for $f$ and $g$ cuspidal automorphic representations on $G L(n) / \mathbf{Q}$, there exists an 
$L$-function denoted $L(f \times g, s)$ with Dirichlet series expansion

$$
\sum_{n \geqslant 1} \lambda_{f \times g}(n) n^{-s}
$$

(say) for $\operatorname{Re}(s)$ large enough, with the properties that:

- We have

$$
\lambda_{f \times g}(p)=\lambda_{f}(p) \lambda_{g}(p)
$$

for all $p$ (see the remark below: This $L(f \times g, s)$ is the unramified Rankin-Selberg convolution).

- The $L$-function $L(f \times g, s)$ extends to a meromorphic function on $\mathbf{C}$ which has no poles except possibly at $s=1$ ([MW]).

- Moreover, it does have a pole at $s=1$ if and only if $g=\bar{f}$ is the contragredient of $f$, so $\lambda_{f \times \bar{f}}(p)=\left|\lambda_{f}(p)\right|^{2}$ for $p \nmid \operatorname{Cond}(f)$.

- The conductor Cond $(f \times g)$ satisfies $([\mathbf{B H}])$

$$
\operatorname{Cond}(f \times g) \leqslant(\operatorname{Cond}(f) \operatorname{Cond}(g))^{n} .
$$

Properly speaking, what we denote here $L(f \times g, s)$ is again the unramified Rankin-Selberg $L$-function, in agreement with our practice with $L(f, s)$. This is why $(2.1)$ holds for all primes $p$. This will be very convenient (see (2.6) below).

The unramified $L$-function $L(f \times g, s)$ differs from the "true" $L$-function by a finite Euler product over $p \mid \operatorname{Cond}(f) \operatorname{Cond}(g)$, which is entire and has no zeros in $\operatorname{Re}(s)>0$ (see Remark 1 and [RS, App. A]).

In the following, we fix a real parameter $z \geqslant 1$ (to be chosen explicitly later), and we let

$$
P=P(z)=\prod_{p<z} p .
$$

Lemma 9. Let $f$ be a cuspidal automorphic representation on $G L(n) / \mathbf{Q}-$ resp. let $f$ and $g$ be cuspidal automorphic representations on $G L(n) / \mathbf{Q}$. For $z$ large enough we have a factorization

$$
L(f, s)=L^{b}(f, s) L^{\sharp}(f, s)
$$

— resp. $L(f \times g, s)=L^{b}(f \times g, s) L^{\sharp}(f \times g, s)$ - with

$$
L^{b}(f, s)=\sum_{(n, P)=1}^{b} \lambda_{f}(n) n^{-s}=\prod_{p \geqslant z}\left(1+\lambda_{f}(p) p^{-s}\right)
$$

- resp.

$$
L^{b}(f \times g, s)=\sum_{(n, P)=1}^{b} \lambda_{f \times g, s}(n) n^{-s}=\prod_{p \geqslant z}\left(1+\lambda_{f}(p) \lambda_{g}(p) p^{-s}\right),
$$


where $L^{\sharp}(f, s)$ - resp. $L^{\sharp}(f \times g, s)$ - is holomorphic and has neither zero nor pole in

$$
\operatorname{Re}(s)>1 / 2 .
$$

Moreover, $L^{\sharp}(f, s)$ is uniformly bounded (in terms of $f$ ) in any region

$$
\operatorname{Re}(s)>1 / 2+\varepsilon
$$

for fixed $\varepsilon>0$ - resp. $L^{\sharp}(f \times g, s)$ is uniformly bounded in terms of $f$ and $g$ in $\operatorname{Re}(s)>1 / 2+\varepsilon$ for any fixed $\varepsilon>0$. Finally, $z$ can be chosen depending only on the family.

Hence we can perform this factorization for all of our $L$-functions and their Rankin-Selberg convolutions.

For a cuspidal automorphic representation $f$ on $G L(n) / \mathbf{Q}$, we let

$$
s(f)=\operatorname{Res}_{s=1} L(f \times \bar{f}, s) .
$$

Since the pole at $s=1$ is simple [MW], this is nonzero.

We also recall the classical convexity bound for the $L$-functions we use.

Lemma 10. Let $f-$ resp. $f$ and $g-$ be as above and for $0 \leqslant \sigma \leqslant 1$, let $\ell(\sigma)$ be the affine function such that

$$
\left\{\begin{array}{l}
\ell(0)=\frac{1}{2} \\
\ell(1)=0,
\end{array}\right.
$$

explicitly $\ell(\sigma)=(1-\sigma) / 2$. For any $\varepsilon>0$, we have

$$
L(f, s) \ll_{\varepsilon}\left(\operatorname{Cond}(f)(|t|+2)^{n}\right)^{\ell(\operatorname{Re}(s))+\varepsilon}
$$

for $0 \leqslant \operatorname{Re}(s) \leqslant 1$, the constant depending only on $\varepsilon$ and the family, and

$$
L(f \times g, s) \ll_{\varepsilon}\left(\operatorname{Cond}(f \times g)(|t|+2)^{n^{2}}\right)^{\ell(\operatorname{Re}(s))+\varepsilon},
$$

for $0 \leqslant \operatorname{Re}(s) \leqslant 1$, the constant depending only on $\varepsilon$ and the family.

Proof. This is well-known. The constant depends only on $\varepsilon$ and the family because of the uniform bound for the Fourier coefficients and the fact that the gamma factors are the same for all $f \in S(q)$.

\section{A mean-value estimate with pseudo-characters.}

Selberg's proof of the analogue of Theorem 2 is based on his notion of pseudocharacters. We will adapt it here to the general case under consideration (such a generalization is not entirely straightforward, compare with $[\mathbf{J}]$ ).

Recall that $z \geqslant 1$ has been fixed in the previous section and $P$ is defined by $(2.3)$. 
Definition. Let $f$ be a cuspidal automorphic representation on $G L(n) / \mathbf{Q}$. For any integer $r \geqslant 1$ with $\lambda_{f}(r) \neq 0$, we let

$$
\psi_{f}(r)=\mu(r) r\left|\lambda_{f}(r)\right|^{-2},
$$

and we define the pseudo-character associated to $f$ and $r$ to be the arithmetic function defined by

$$
\psi_{f, r}(n)=\mu(n)^{2} \psi_{f}((n, r)) .
$$

Because of its appearance in the denominator, we have to work with integers for which $\left|\lambda_{f}(r)\right|$ is not too small. Specifically, let $\delta, 0<\delta<1 / 2$, be a parameter to be chosen later, and define

$$
\begin{aligned}
& R(f)=\{r \geqslant 1 \mid r \text { is squarefree, }(r, \operatorname{Cond}(f) P)=1, \text { and } \\
& \left.\qquad p|r \Rightarrow| \lambda_{f}(p) \mid>p^{-\delta}\right\} .
\end{aligned}
$$

Such integers are quite abundant, as the next lemma shows.

Lemma 11. Let $f \in S(q)$. We have

$$
\sum_{\substack{r \leqslant R \\ r \in R(f)}} \frac{1}{\left|\psi_{f}(r)\right|} \gg s(f) \log R
$$

for $R>q^{C}$, where $C$ is any constant $>n A$. The implied constant depends only on the family once $C$ is chosen.

Moreover

$$
\sum_{\substack{r \leqslant R \\ r \in R(f)}} \frac{1}{\left|\psi_{f}(r)\right|} \ll s(f) \log R,
$$

for $R \geqslant 2$, the constant depending only on the family.

Proof. This is a consequence of the properties of the Rankin-Selberg convolution and is very similar to what will be done in the next section. Remark that

$$
\frac{1}{\left|\psi_{f}(r)\right|}=\frac{\left|\lambda_{f}(r)\right|^{2}}{r}=\frac{\lambda_{f \times \bar{f}}(r)}{r} .
$$

One computes

$$
\sum_{r \in R(f)} \lambda_{f \times \bar{f}}(r) \varphi\left(\frac{r}{R}\right)
$$

(where $\varphi$ is a suitable test function) using Mellin transform. For $\operatorname{Re}(s)>$ $1-2 \delta$, one can replace $L(f \times \bar{f}, s)$ by the product over the primes in $R(f)$, since

$$
\prod_{p}\left(1+p^{-2 \delta-s}\right)
$$


converges absolutely in that range. Shifting the contour to $\operatorname{Re}(s)=1-2 \delta+\varepsilon$ and estimating $L(f \times \bar{f}, s)$ on that line using (2.2) and Lemma 10 yields the result.

For (3.5), we simply write by positivity

$$
\sum_{\substack{r \leqslant R \\ r \in R(f)}} \frac{1}{\left|\psi_{f}(r)\right|} \leqslant \sum_{r \leqslant R} \frac{\lambda_{f \times \bar{f}}(r)}{r}
$$

so we need no assumption on $R$.

Now we have the following formal lemma about pseudo-characters (see $[\mathbf{J}$, Lemma 2-3]), which incorporates their orthogonality.

Lemma 12. Let $f$ and $g$ be cuspidal automorphic representations on $G L(n) / \mathbf{Q}, r \in R(f), t \in R(g)$. Let $h$ be the arithmetic function defined by the Dirichlet polynomial identity

$$
\begin{aligned}
\sum_{d \geqslant 1} h(d) d^{-s}= & \prod_{\substack{p \mid r \\
p \nmid t}}\left(1+\left(\psi_{f}(p)-1\right) p^{-s}\right) \prod_{\substack{p \mid t \\
p \nmid r}}\left(1+\left(\psi_{g}(p)-1\right) p^{-s}\right) \\
& \cdot \prod_{p \mid(r, t)}\left(1+\left(\psi_{f}(p) \psi_{g}(p)-1\right) p^{-s}\right) .
\end{aligned}
$$

Then we have

$$
\psi_{f, r}(n) \psi_{g, t}(n)=\mu(n)^{2} \sum_{d \mid n} h(d)
$$

for all $n \geqslant 1$. If $g=\bar{f}$, we have

$$
\sum_{d \geqslant 1} h(d)\left|\lambda_{f}(d)\right|^{2} \rho_{f}(d) d^{-1}=\delta(r, t)\left|\psi_{f}(r)\right|,
$$

where

$$
\rho_{f}(d)=\prod_{p \mid d}\left(1+\left|\lambda_{f}(p)\right|^{2} p^{-1}\right)^{-1} .
$$

In particular, note that, by definition, $h$ is supported on the set of divisors of $r t$.

The next result can be seen as an analogue with pseudo-characters of the general mean-value estimate of [DK, §4].

Proposition 13. Assume that $N \geqslant R^{2}$ and that there exists $\varepsilon>0$ such that

$$
N^{1 / 2-\varepsilon}>q^{d+n A / 2} R^{2+2 \delta}(\log R)
$$


Then we have

$$
\sum_{f \in S(q)} \frac{1}{s(f)} \sum_{\substack{r \leqslant R \\ r \in R(f)}} \frac{1}{\left|\psi_{f}(r)\right|}\left|\sum_{n \sim N} a_{n} \psi_{f, r}(n) \lambda_{f}(n)\right|^{2} \ll N \sum_{n \sim N}\left|a_{n}\right|^{2}
$$

for any complex numbers $a_{n}$. The implied constant depends on the chosen $\varepsilon$ and on the family only.

From this, proceeding as in [M, VII], one deduces:

Corollary 14. For any $f \in S(q)$, let $J(f) \subset M(\alpha, T)$ a finite set of wellspaced points, i.e.,

$$
\operatorname{Im}\left(\rho-\rho^{\prime}\right) \geqslant \frac{1}{\log q}
$$

for $\rho \neq \rho^{\prime} \in J(f)$. Then, under the same assumptions as above, we have

$$
\begin{aligned}
& \sum_{f \in S(q)} \frac{1}{s(f)} \sum_{\rho \in J(f)} \sum_{\substack{r \leqslant R \\
r \in R(f)}} \frac{1}{\left|\psi_{f}(r)\right|}\left|\sum_{n \sim N} a_{n} \psi_{f, r}(n) \lambda_{f}(n) n^{-\rho}\right|^{2} \\
& \ll T^{B} \log (q N)\left(1+\log \frac{\log 2 N}{\log 2 R}\right) \sum_{n \sim N}\left|a_{n}\right|^{2} n^{1-2 \alpha}
\end{aligned}
$$

for any complex numbers $a_{n}$, for some constant $B>0$ depending only on the family.

Proof of the proposition. (Compare [DK, §4].)

We use duality and smooth the sum by positivity so we consider instead the sum

$$
\begin{aligned}
& \sum_{n \sim N}\left|\sum_{f \in S(q)} \frac{1}{\sqrt{s(f)}} \sum_{\substack{r \leqslant R \\
r \in R(f)}} \frac{b(f, r)}{\sqrt{\psi_{f}(r)}} \lambda_{f}(n) \psi_{f, r}(n)\right|^{2} \\
& \leqslant \sum_{f, g \in S(q)} \sum_{\substack{r, t \leqslant R \\
r \in R(f) \\
t \in R(g)}} \frac{b(f, r) \overline{b(g, t)}}{\sqrt{s(f) s(g) \mid \psi_{f}(r) \psi_{g}(t)}} S_{1}(f, g, r, t)
\end{aligned}
$$

where

$$
S_{1}=\sum_{n \geqslant 1} \psi_{f, r}(n) \overline{\psi_{g, t}(n)} \lambda_{f}(n) \overline{\lambda_{g}(n)} \varphi\left(\frac{n}{N}\right)
$$

for some fixed smooth test function $\varphi:[0,+\infty[\longrightarrow[0,1]$ with compact support in $[1 / 2,3]$ such that $\varphi(x)=1$ for $1 \leqslant x \leqslant 2$. 
By (3.7), we have

$$
\begin{aligned}
S_{1}= & \sum_{\substack{d \leqslant N \\
(d, P)=1}}^{b} h(d) \lambda_{f}(d) \overline{\lambda_{g}(d)} \sum_{\substack{(n, d)=1 \\
(n, P)=1}}^{b} \lambda_{f}(n) \overline{\lambda_{g}(n)} \varphi\left(\frac{n d}{N}\right) \\
= & \sum_{\substack{d \leqslant N \\
(d, P)=1}}^{b} h(d) \lambda_{f}(d) \overline{\lambda_{g}(d)} T_{d}(N) \quad \text { (say) }
\end{aligned}
$$

(we have used the fact that for $n, d$ with $n d$ in the support of the pseudocharacters we have $\lambda_{f}(n d)=\lambda_{f}(n) \lambda_{f}(d)$, and similarly for $g$ ).

We now let

$$
\begin{aligned}
L_{d}^{b}(f \times \bar{g}, s) & =\sum_{\substack{(n, d)=1 \\
(n, P)=1}}^{b} \lambda_{f}(n) \overline{\lambda_{g}(n)} n^{-s} \\
& =L^{b}(f \times \bar{g}, s) \prod_{\substack{p \mid d \\
p \geqslant z}}\left(1+\lambda_{f}(p) \overline{\lambda_{g}(p)} p^{-s}\right)^{-1}
\end{aligned}
$$

(see Lemma 9). By Mellin transform we can write

$$
T_{d}(N)=\frac{1}{2 i \pi} \int L_{d}^{b}(f \times \bar{g}, s) \hat{\varphi}(s)(N / d)^{s} d s .
$$

By Lemma 9, we can shift the contour up to $\operatorname{Re}(s)=1 / 2+\varepsilon$, for any $\varepsilon>0$, and from the properties of the Rankin-Selberg convolution recalled before the lemma, we encounter on the way only one possible pole at $s=1$ at $s=1$, which occurs if and only if $f=g$. On $\operatorname{Re}(s)=1 / 2+\varepsilon$, we estimate the $L$-function using the factorization and the convexity bound (2.9) for $L(f \times \bar{g}, s)$. By $[\mathbf{B H}]$, we have

$$
\operatorname{Cond}(f \times g) \leqslant(\operatorname{Cond}(f) \operatorname{Cond}(g))^{n} \leqslant q^{2 n A},
$$

hence for any $\varepsilon>0$ we have

$$
L_{d}^{b}(f \times \bar{g}, s) \ll_{\varepsilon} q^{n A / 2} d^{\varepsilon} T^{B}
$$

for $\operatorname{Re}(s)=1 / 2+\varepsilon$, for some $B>0$. It follows that

$$
T_{d}(N)=\delta(f, g) s_{d}(f) \frac{N}{d}+\underline{O_{\varepsilon}}\left(N^{1 / 2+\varepsilon} d^{-1 / 2+\varepsilon} q^{n A / 2}\right)
$$

for all $N$ and $d$, where

$$
s_{d}(f)=\operatorname{Res}_{s=1} L_{d}^{b}(f \times \bar{f}, s)=s(f) \rho_{f}(d) L^{\sharp}(f \times \bar{f}, 1) .
$$


The implied constant in (3.15) depends on $z, \varepsilon$ and the family. For fixed $z$, we remark that

$$
L^{\sharp}(f \times \bar{f}, 1) \ll 1
$$

uniformly for all $f$ in the family.

Summing over $d$ in (3.14), the error terms contribute at most

$$
N^{1 / 2+\varepsilon} q^{n A / 2} \sum_{\substack{d \leqslant N \\(d, P)=1}}^{b} \frac{\left|h(d) \lambda_{f}(d) \lambda_{g}(d)\right|}{\sqrt{d}} .
$$

But by multiplicativity, we have

$$
\begin{aligned}
& \sum_{\substack{d \leqslant N \\
(d, P)=1}}^{b} \frac{\left|h(d) \lambda_{f}(d) \lambda_{g}(d)\right|}{\sqrt{d}} \\
& \ll \prod_{p \mid r}\left(1+\frac{2\left|\psi_{f}(p) \lambda_{f}(p)\right|}{p^{1 / 2}}\right) \prod_{p \mid t}\left(1+\frac{2\left|\psi_{g}(p) \lambda_{g}(p)\right|}{p^{1 / 2}}\right) \\
& \ll_{\varepsilon} R^{2+2 \delta+\varepsilon} N^{1 / 2+\varepsilon} q^{n A / 2} .
\end{aligned}
$$

The main term contributes only if $f=g$, and is then (because $N \geqslant R^{2} \geqslant$ $r t$ so the sum over $d$ can be extended to all integers), equal to

$$
\begin{aligned}
& s(f) L^{\sharp}(f \times \bar{f}, 1) N \sum_{\substack{d \leqslant N \\
(d, P)=1}}^{b} h(d) \rho_{f}(d)\left|\lambda_{f}(d)\right|^{2} d^{-1} \\
& =s(f) L^{\sharp}(f \times \bar{f}, 1) N \sum_{\substack{d \geqslant 1 \\
(d, P)=1}}^{b} h(d) \rho_{f}(d)\left|\lambda_{f}(d)\right|^{2} d^{-1} \\
& =\delta(r, t) s(f)\left|\psi_{f}(r)\right| L^{\sharp}(f \times \bar{f}, 1) N \text { by }(3.8) .
\end{aligned}
$$

In (3.12), the "diagonal contribution" is therefore equal to

$$
N \sum_{f \in S(q)} L^{\sharp}(f \times \bar{f}, 1) \sum_{\substack{r \leqslant R \\ r \in R(f)}}|b(r, f)|^{2} \ll N \sum_{f \in S(q)} \sum_{\substack{r \leqslant R \\ r \in R(f)}}|b(r, f)|^{2} \text { (by (3.16)), }
$$

the implied constant depending only on the family.

On the other hand, the error terms contribute

$$
R^{2+2 \delta} N^{1 / 2+\varepsilon} q^{n A / 2}\left(\sum_{f \in S(q)} \frac{1}{\sqrt{s(f)}} \sum_{\substack{r \leqslant R \\ r \in R(f)}} \frac{b(f, r)}{\sqrt{\left|\psi_{f}(r)\right|}}\right)^{2}
$$


and by Cauchy's inequality, the right-hand sum is

$$
\begin{aligned}
& \left(\sum_{f \in S(q)} \frac{1}{\sqrt{s(f)}} \sum_{\substack{r \leqslant R \\
r \in R(f)}} \frac{b(f, r)}{\sqrt{\left|\psi_{f}(r)\right|}}\right)^{2} \\
& \leqslant\left(\sum_{f \in S(q)} \sum_{\substack{r \leqslant R \\
r \in R(f)}}|b(r, f)|^{2}\right)\left(\sum_{f \in S(q)} \frac{1}{s(f)} \sum_{\substack{r \leqslant R \\
r \in R(f)}} \frac{1}{\left|\psi_{f}(r)\right|}\right) \\
& \ll|S(q)|(\log R) \sum_{f \in S(q)} \sum_{\substack{r \leqslant R \\
r \in R(f)}}|b(r, f)|^{2}(\text { by }(3.5)),
\end{aligned}
$$

the implied constant depending only on the family.

Hence the norm of the quadratic form is bounded by

$$
N+N^{1 / 2+\varepsilon} q^{n A / 2+d} R^{2+2 \delta}(\log R),
$$

and the assumption (3.10) proves the proposition.

\section{End of the proof.}

We finish the proof essentially as in $[\mathbf{J}]$. Let $1 \leqslant w<y$ be two parameters, to be chosen later. Define

$$
m(d)= \begin{cases}1 & \text { if } d \leqslant w, \\ \frac{\log (d / y)}{\log (w / y)} & \text { if } w \leqslant d \leqslant y, \\ 0 & \text { if } y<d\end{cases}
$$

and

$$
\lambda_{d}=\mu(d) m(d)
$$

(the Selberg weights). Define also

$$
\Delta(n)=\sum_{d \mid n} \lambda_{d}
$$

Notice that

$$
\Delta(1)=1, \quad \Delta(n)=0 \text { if } 1<n \leqslant w .
$$

We will need the following result of Graham [G, Lemma 9]:

Lemma 15. For any $\alpha$ with $1 / 2<\alpha<1$, we have

$$
\sum_{n \leqslant x} \Delta(n)^{2} n^{1-2 \alpha} \ll \frac{\log (x / w)}{\log (y / w)} x^{2-2 \alpha}
$$

for all $x \geqslant 1$, with an absolute implied constant. 
We now introduce the mollifier.

Lemma 16. Let $f \in S(q), r \in R(f)$. We have the identity

$$
\sum_{(n, P)=1}^{b} \Delta(n) \psi_{f, r}(n) \lambda_{f}(n) n^{-s}=L^{b}(f, s) M_{r}(f, s)
$$

where

$$
M_{r}(f, s)=\sum_{d \geqslant 1} \lambda_{d} \eta_{f, r}(d ; s) \psi_{f, r}(d) \lambda_{f}(d) d^{-s}
$$

and

$$
\eta_{f, r}(d ; s)=\prod_{p \mid r /(r, d)}\left(1+\psi_{f}(p) \lambda_{f}(p) p^{-s}\right) \prod_{p \mid r d}\left(1+\lambda_{f}(p) p^{-s}\right)^{-1} .
$$

Proof. This is a formal identity using the multiplicativity of $\psi_{f, r}(n)$ and $\lambda_{f}(n)$ : The left-hand side of (4.3) is

$$
\begin{aligned}
& \sum_{(n, P)=1}^{b} \Delta(n) \psi_{f, r}(n) \lambda_{f}(n) n^{-s} \\
= & \sum_{(d, P)=1} \lambda_{d} \psi_{f, r}(d) \lambda_{f}(d) d^{-s} \sum_{(n, d P)=1}^{b} \psi_{f, r}(n) \lambda_{f}(n) n^{-s} \\
= & \sum_{(d, P)=1} \lambda_{d} \psi_{f, r}(d) \lambda_{f}(d) d^{-s} \prod_{(p, d P)=1}\left(1+\psi_{f, r}(p) \lambda_{f}(p) p^{-s}\right) \\
= & L^{b}(f, s) \prod_{p \mid r} \frac{1+\psi_{f}(p) \lambda_{f}(p) p^{-s}}{1+\lambda_{f}(p) p^{-s}} \sum_{(d, P)=1} \lambda_{d} \psi_{f, r}(d) \lambda_{f}(d) d^{-s} \\
& \cdot \prod_{p \mid(r, d)}\left(1+\psi_{f}(p) \lambda_{f}(p) p^{-s}\right) \prod_{p \mid d /(r, d)}\left(1+\lambda_{f}(p) p^{-s}\right) \\
= & L^{b}(f, s) M_{r}(f, s) .
\end{aligned}
$$

Lemma 17. Let $f \in S(q), r \in R(f)$. If $z$ is chosen large enough, then for any $s \in \mathbf{C}$ with $0<\operatorname{Re}(s) \leqslant 1$, we have

$$
M_{r}(f, s) \ll_{\varepsilon} r^{1+2 \delta-\operatorname{Re}(s)+\varepsilon} y^{1-\sigma+\varepsilon}
$$

where the constant depends only on $\varepsilon$ and the family.

Proof. We have $\left|\lambda_{d}\right| \leqslant 1$ for $d \leqslant y$ and $\lambda_{d}=0$ for $d>y$. Since $r \in R(f)$, we also have

$$
\left|\psi_{f, r}(d) \lambda_{f}(d)\right| \leqslant(r, d)^{1+\delta} \leqslant r^{\delta}(r, d) .
$$


Again since $r \in R(f)$, and since $\operatorname{Re}(s) \leqslant 1$, we have

$$
\left|\prod_{p \mid r /(r, d)}\left(1+\psi_{f}(p) \lambda_{f}(p) p^{-s}\right)\right| \leqslant \tau(r) r^{1+\delta-\operatorname{Re}(s)} .
$$

Finally, since $\operatorname{Re}(s)>0$ we have

$$
\left|1+\lambda_{f}(p) p^{-s}\right| \geqslant 1-p^{-\sigma} \geqslant 1 / 2
$$

if $p \geqslant z$ and $z$ is large enough, so

$$
\left|\prod_{r \mid r d}\left(1+\lambda_{f}(p) p^{-s}\right)^{-1}\right| \leqslant \tau(r d) .
$$

The result now follows from the definition of $M_{r}(f, s)$.

Lemma 18. Let $f \in S(q), r \in R(f)$ with $r \leqslant R$. Let $T \geqslant 1$ be a real number and let $\alpha$ be such that

$$
1 / 2 \leqslant \alpha \leqslant 1 \text {. }
$$

Let $X \geqslant 1$ be a parameter, $x=X(\log q T)^{2}$, and let $z_{r}(f, s)$ be the "zerodetector"

$$
z_{r}(f, s)=\sum_{\substack{1<n<x \\(n, P)=1}}^{b} \Delta(n) \psi_{f, r}(n) e^{-n / X} \lambda_{f}(n) n^{-s} .
$$

Last, let $\rho \in M(\alpha, T)$ be a zero of $L(f, s)$.

Fix $\varepsilon>0$. Then we have

$$
1 \ll_{\varepsilon, \alpha} z_{r}(f, \rho)
$$

provided that the following inequalities hold:

$$
\left\{\begin{array}{l}
X \geqslant\left(y q^{1 / 2} T^{n / 2} R^{1+4 \delta}\right)^{1 /(2 \alpha-1)+\varepsilon} \\
\log X \ll \log q T \\
(\log q)^{1 / 2} \leqslant \log R \leqslant \frac{1}{2} \log x .
\end{array}\right.
$$

The implied constant depends only on $\varepsilon, \alpha$ and the family.

Proof. This is again a Mellin transform argument. Let $\sigma$ be any real number with $\sigma+\alpha>1 / 2$, say $\sigma=1 / 2-\alpha+\varepsilon^{\prime}$ with $\varepsilon^{\prime}>0$. We have

$$
\begin{aligned}
& e^{-1 / X}+\sum_{\substack{n>1 \\
(n, P)=1}}^{b} \Delta(n) \psi_{f, r}(n) e^{-n / X} \lambda_{f}(n) n^{-\rho} \\
& =\int_{(3)} L^{b}(f, s+\rho) M_{r}(f, s+\rho) \Gamma(s) X^{s} d s \\
& =\int_{(\sigma)} L^{b}(f, s+\rho) M_{r}(f, s+\rho) \Gamma(s) X^{s} d s
\end{aligned}
$$


by contour shift, since the zero of $L^{\mathrm{b}}(f, s+\rho)$ cancels the simple pole of $\Gamma(s)$. On the line $\operatorname{Re}(s)=\sigma$, we have $\operatorname{Re}(s+\rho)=\sigma+\operatorname{Re}(\rho)>1 / 2+\varepsilon^{\prime}$, so we can apply Lemma 9 and Lemma 10 to estimate $L^{b}(f, s+\rho)$.

Estimating the mollifier using Lemma 17, and the fact that $\operatorname{Re}(s) \geqslant \alpha$, we see that the integral on $\operatorname{Re}(s)=\sigma$ is

$$
\ll_{\varepsilon} X^{1 / 2-\alpha+\varepsilon} R^{1 / 2+2 \delta+\varepsilon} y^{1 / 2+\varepsilon} q^{\frac{1}{4}+\varepsilon} T^{\frac{n}{4}+\varepsilon} .
$$

The left-hand side of $(4.5)$ is $z_{r}(f, \rho)+\underline{O}_{\varepsilon}(1)$, so the result follows.

To conclude the proof, suppose given for every $f \in S(q)$ a subset $Z(f)$ of well-spaced points as in Corollary 14, and let $Z$ be the union of the $Z(f)$. Consider the sum

$$
Z^{\prime}=\sum_{f \in S(q)} \sum_{\substack{r \leqslant R \\ r \in R(f)}} \frac{|Z(f)|}{s(f)\left|\psi_{f}(r)\right|}
$$

Lemma 19. Assume that $R>q^{C}$ where $C>n A$. We have

$$
Z^{\prime} \gg(\log R)|Z|
$$

where the implied constant depends only on the family.

Proof. This is clear from (3.4), by summing first over $r$ :

$$
Z^{\prime}=\sum_{f \in S(q)} \frac{|Z(f)|}{s(f)} \sum_{\substack{r \leqslant R \\ r \in R(f)}} \frac{1}{\left|\psi_{f}(r)\right|} \gg(\log R) \sum_{f \in S(q)}|Z(f)| .
$$

Lemma 20. We have

$$
Z^{\prime} \ll T^{B}(\log q) x^{2(1-\alpha)}
$$

if the parameters $\alpha, w, y, R$, satisfy the assumptions (3.10) (with $N=x$ ) and (4.4) and if

$$
\log x \ll \log (y / w) .
$$

The implied constant depends only on the family.

Proof. We have by Lemma 18

$$
Z^{\prime} \ll \sum_{f \in S(q)} \frac{1}{s(f)} \sum_{\rho \in Z(f)} \sum_{\substack{r \leqslant R \\ r \in R(f)}} \frac{1}{\left|\psi_{f}(r)\right|}\left|z_{r}(f, \rho)\right|^{2} .
$$

This expression if of the type considered in Corollary 14 above, with coefficients

$$
a_{n}=\Delta(n) e^{-n / X} \text { for } 1<n<x
$$


Since $a_{n}=0$ for $1<n \leqslant w$, we find

$$
\begin{aligned}
Z^{\prime} & \ll T^{B}(\log q) \sum_{w<n<x} \Delta(n)^{2} n^{1-2 \alpha} \\
& \left.\ll T^{B}(\log q) x^{2(1-\alpha)} \quad \text { (by Lemma } 15\right) .
\end{aligned}
$$

Theorem 2 follows now easily: To count the zeros of $L(f, s)$, we partition $M(\alpha, T)$ in rectangles

$$
R_{k}=[\alpha, 1] \times[k /(\log q),(k+1) /(\log q)] .
$$

Fixing a parity of $k$ (even or odd), for each $f$ and $k$ pick (if it exists) a zero $\rho_{f, k}$ of $L(f, s)$ in $R_{k}$. The resulting sets $Z(f)$ are then well-spaced (for each parity of $k$ separately).

By comparing Lemma 19 and Lemma 20, we find that (if the assumptions are satisfied), we have

$$
\sum_{f \in S(q)}|Z(f)| \ll T^{B} x^{2(1-\alpha)}
$$

if $R$ and $q$ are logarithmically comparable.

To take all zeros of $L(f, s)$ into account, we use the following simple density lemma (see [J, Lemma 8]) originally proved by Linnik.

Lemma 21. The number of zeros of $L(f, s)$ in the square

$$
\left\{s \in \mathbf{C}|\alpha \leqslant \operatorname{Re}(s) \leqslant 1, \quad| \operatorname{Im}(s)-t \mid \leqslant \frac{1}{2}(1-\alpha)\right\}
$$

is

$$
\ll(1-\alpha) \log (q(|t|+2))+1,
$$

the implied constant depending only on the family.

Proof. The proof is the same as in $[\mathbf{P}$, p. 332] note that Prachar gives a bound

$$
\ll(1-\alpha) \log (q(|t|+2))
$$

subject to $\alpha \geqslant 1-1 /(\log q)$. However, one can drop that condition by adding the extra +1 as in (4.6).

It remains to choose the parameters to obtain Theorem 2. For this, write

$$
R=q^{a_{1}}, \quad w=q^{a_{2}}, \quad y=q^{a_{3}}, \quad X=q^{a_{4}}
$$

and let $a_{5}=(2 \alpha-1) a_{4}$.

We let $\delta \rightarrow 0$, and take

$$
a_{1} \downarrow n A, \quad a_{2}=1, \quad a_{3} \downarrow 1, \quad a_{5} \downarrow \frac{5 n A}{2}+d,
$$


hence we obtain the value of $c_{0}^{\prime}$ in (1.5). Easy computations show that all conditions are satisfied (if $\alpha \geqslant 3 / 4$ ).

\section{A density theorem for $L$-functions in families of elliptic curves.}

In this section we prove Corollary 8 .

Let $E_{t}$ be the one parameter familly of elliptic curves given by Equation (1.8). Recall that we assume that the $j$-invariant $j(t) \in \mathbf{Q}(t)$ is nonconstant.

For any $x \in \mathbf{Z}$ such that $\Delta(x) \neq 0$, the specialized fiber $E_{x}$ is an elliptic curve and we note $N_{x}$ his conductor.

From the work of Wiles, Taylor-Wiles, Breuil-Conrad-Diamond-Taylor ([Wi, TW, Di, BCDT] $]$ ) and from the Gelbart-Jacquet lift $[\mathbf{G J}]$, there exist automorphic forms $f_{x}$ and $\operatorname{sym}^{(2)}\left(f_{x}\right)$ on $G L(2) / \mathbf{Q}$ and $G L(3) / \mathbf{Q}$ (respectively) with (analytically normalized) $L$-functions equal to the Hasse-Weil $L$-function of $E_{x}$ and its symmetric square.

Moreover, the conductor of $f_{x}$ is $=N_{x}$ and the conductor $M_{x}$ of $\operatorname{sym}^{(2)} f_{x}$ is a divisor of $N_{x}^{2}$. We have therefore

$$
N_{x} \leqslant|\Delta(x)| \ll(1+|x|)^{\operatorname{deg}(\Delta)}, M_{x} \ll(1+|x|)^{2 \operatorname{deg}(\Delta)},
$$

with implied constants depending only on the elliptic curve $E_{t} / \mathbf{Q}(t)$.

There might be a multiplicity involved, different $x$ giving the same automorphic form.

Lemma 22. There exist a constant $C>0$ depending only on the equation for $E_{t} / \mathbf{Q}(t)$ such that the number of $x$ with the same $f_{x}$ is $\leqslant C$ and the number of $x$ with the same $\operatorname{sym}^{(2)} f_{x}$ is $\leqslant C$.

Proof. Let $E=E_{x}$ one of the fibers. From the isogeny theorem [Fa], $L\left(E_{x}, s\right)=L\left(E_{y}, s\right)$ if and only if $E_{x} \simeq E_{y}$, where this indicates isogeny over $\mathbf{Q}$. By a result of Mazur [Ma], the number of elliptic curves over $\mathbf{Q}$ in a given isogeny class is $\leqslant D$ for some absolute constant $D>0$. Hence all the $j(y)$ with $L\left(E_{y}, s\right)=L\left(E_{x}, s\right)$ belong to a finite set. Since $j$ is nonconstant, each $j$ has at most $\operatorname{deg}(j)$ preimages.

For the symmetric square, we have (see Ramakrishnan's Appendix to [DK] $) L\left(\operatorname{sym}^{(2)} f_{x}, s\right)=L\left(\operatorname{sym}^{(2)} f_{y}, s\right)$ if and only if $f_{y}$ is a quadratic twist of $f_{x}$. In such a case, $j(y)=j(x)\left(E_{x}\right.$ and $E_{y}$ are isomorphic over a quadratic field), hence the argument above goes through also.

Now the corollary follow immediately from Theorem 2 applied to the families

$$
\begin{aligned}
S(q) & =\left\{f_{x}|| x \mid \leqslant q\right\} \text { with } d=1, n=2, A=\operatorname{deg}(\Delta) \\
S^{2}(q) & =\left\{\operatorname{sym}^{(2)} f_{x}|| x \mid \leqslant q\right\} \text { with } d=1, n=3, A=2 \operatorname{deg}(\Delta),
\end{aligned}
$$


(strictly speaking we have to exclude in the last case the values of $x$ such that $E_{x}$ is $\mathrm{CM}$, in which case the symmetric square is not a cusp form; however this happens only in finitely many cases, since $j(x)$ has to belong to a finite set, and one can add up the corresponding trivial estimate for the number of zeros).

\section{References}

[Bo] E. Bombieri, Le grand crible dans la théorie analytique des nombres, Astérisque, 18, SMF, 1974, MR 51 \#8057, Zbl 0618.10042.

[BC] A. Borel and W. Casselman, Automorphic Forms, Representations, and LFunctions, Proc. Symp. Pure Math. XXXIII, Vol. 1 and 2, A.M.S., 1979, MR 80g:10002a, Zbl 0403.00002; MR 80g:10002b, Zbl 0403.00003.

[BCDT] C. Breuil, B. Conrad, F. Diamond and R. Taylor, On the modularity of elliptic curves over Q, J. Amer. Math. Soc., 14(4) (2001), 843-939 (electronic), MR 2002d:11058, Zbl 0982.11033.

[Bu] D. Bump, Automorphic Forms and Representations, Cambridge Studies in Advanced Mathematics, 55, Cambridge University Press, 1997, MR 97k:11080, Zbl 0868.11022.

[BH] C.J. Bushnell and G. Henniart, An upper bound on conductors for pairs, J. Number Theory, 65(2) (1997), 183-196, MR 98h:11153, Zbl 0884.11049.

[C] D. Cox, Primes of the Form $x^{2}+n y^{2}$, Wiley, 1989, MR 90m:11016, Zbl 0701.11001.

[Di] F. Diamond, On deformation rings and Hecke rings, Annals of Math., 144 (1996), 137-166, MR 97d:11172, Zbl 0867.11032.

[DK] W. Duke and E. Kowalski, A problem of Linnik for elliptic curves and meanvalue estimates for automorphic representations. With an appendix by Dinakar Ramakrishnan, Inventiones Math., 139 (2000), 1-39, MR 2001b:11034.

[Fa] G. Faltings, Endlichkeitssätze für abelsche varietäten über zahlkörpern, Inventiones Math., 73(3) (1983), 349-366, MR 85g:11026a, Zbl 0588.14026.

[Fo] E. Fogels, On the zeros of L-functions, Acta Arith., 11 (1965), 67-96, MR 31 \#1230, Zbl 0136.03004.

[GJ] S. Gelbart and H. Jacquet, A relation between automorphic representations of $G L(2)$ and $G L(3)$, Ann. Sci. E.N.S. 4ème série, 11(4) (1978), 471-552, MR 81e:10025, Zbl 0406.10022.

[G] S. Graham, On Linnik's constant, Acta. Arith., 39 (1981), 163-179, MR 83d:10050, Zbl 0464.10032.

[GS] A. Granville and K. Soundararajan, The distribution of value of $L(1, \chi)$. Preprint, 2001.

[Iw1] H. Iwaniec, Topics in Classical Automorphic Forms, Grad. Studies in Math., 17, A.M.S., 1997, MR 98e:110, Zbl 0905.11023.

[JPS] H. Jacquet, I.I. Piatetskii-Shapiro and J.A. Shalika, Rankin-Selberg convolutions, Amer. Jour. of Math., 105 (1983), 367-464, MR 85g:11044, Zbl 0525.22018.

[J] M. Jutila, On Linnik's constant, Math. Scand., 41 (1967), 45-62, MR 57 \#16230, Zbl 0363.10026.

[KS] H. Kim and P. Sarnak, Appendix to Functoriality for the exterior square of $\mathrm{GL}_{4}$ and symmetric fourth of $\mathrm{GL}_{2}$ by $\mathrm{H}$. Kim. Preprint, 2000. 
[KM1] E. Kowalski and P. Michel, Sur les zéros des fonctions L automorphes de grand niveau. Préprint de l'Université d'Orsay, 54, 1997.

[KM2] The analytic rank of $J_{0}(q)$ and zeros of automorphic L-functions, Duke Math. J., 100 (1999), 503-542, MR 2001b:11060.

[Li1] Yu.V. Linnik, On the least prime in an arithmetic progression, I, Rec. Math (Mat. Sbornik) N.S., 15 (57) (1944), 139-178, MR 6,260b, Zbl 0063.03584.

[Li2] - On the least prime in an arithmetic progression, II, Rec. Math (Mat. Sbornik) N.S., 15 (57) (1944), 347-368, MR 6,260c, Zbl 0063.03585.

$[\mathrm{Lu}] \quad$ W. Luo, Values of symmetric L-functions at 1, J. Reine Angew. Math., 506 (1999), 215-235, MR 2001d:11055, Zbl 0969.11018.

[Ma] B. Mazur, Rational isogenies of prime degree, Invent. Math., 44 (1978), 129-162, MR 80h:14022, Zbl 0386.14009.

[Mi] T. Miyake, Modular Forms, Springer Verlag, 1989, MR 90m:11062, Zbl 0701.11014.

[MW] C. Moeglin and J.L. Waldspurger, Pôles des fonctions L de paires pour $G L(N)$, appendix to 'Le spectre résiduel de $G L(n)$ ', Ann. Sci. ENS (4ème série), 22 (1989), 605-674, MR 91b:22028, Zbl 0696.10023.

[M] H.L. Montgomery, Topics in Multiplicative Number Theory, Lecture Notes in Math., 227, Springer-Verlag, 1971, MR 49 \#2616, Zbl 0216.03501.

[MV] H.L. Montgomery and R. Vaughan, Extreme values of Dirichlet L-functions at 1, in 'Number Theory in Progress' (eds. K. Gyory, H. Iwaniec and J. Urbanowicz), de Gruyter, Berlin, 1999, 1039-1052, MR 2000m:11075, Zbl 0942.11040.

[P] K. Prachar, Primzahlverteilung, Grundl. der Math. Wiss., Springer-Verlag, 1957, MR 19,393b, Zbl 0080.25901.

[Ro] E. Royer, Statistique de la variable $L\left(\operatorname{sym}^{(2)} f, 1\right)$, Math. Ann., 321(3) (2001), 667-687, CMP 1871974.

[RS] Z. Rudnick and P. Sarnak, Zeros of principal L-functions and random matrix theory, Duke Math. J., 81 (1996), 269-322, MR 97f:11074, Zbl 0866.11050.

[TW] R. Taylor and A. Wiles, Ring theoretic properties of certain Hecke algebras, Annals of Math., 141(3) (1995), 553-572, MR 96d:11072, Zbl 0823.11030.

[We] A. Weiss, The least prime ideal, J. Reine Angew. Math., 338 (1983), 56-94, MR 84d:12011, Zbl 0492.12008.

[Wi] A. Wiles, Modular elliptic curves and Fermat's last theorem, Annals of Math., 141(3) (1995), 443-551, MR 96d:11071, Zbl 0823.11029.

Received April 9, 2001 and revised July 30, 2001.

Université BordeAux I - A2X

351, COURS DE LA LibÉRATION

33405 Talence Cedex

FRANCE

E-mail address: emmanuel.kowalski@math.u-bordeaux.fr

CC 051, MAThÉMATiques

Université Montpellier II

Place Eugène Bataillon

34095 Montpellier, Cedex 5

FRANCE

E-mail address: michel@darboux.math.univ-montp2.fr 